\title{
Noncommutative field theory on rank one symmetric spaces
}

\author{
Pierre Bieliavsky, Razvan Gurau, and Vincent Rivasseau
}

\begin{abstract}
Quantum field theory has been shown recently renormalizable on flat Moyal space and better behaved than on ordinary space-time. Some models at least should be completely finite, even beyond perturbation theory. In this article a first step is taken to extend such theories to non-flat backgrounds such as solvable symmetric spaces.
\end{abstract}

Mathematics Subject Classification (2000). 81T20, 81T75.

Keywords. Quantum field theory, symmetric spaces, noncommutative geometry.

\section{Introduction}

Following the pioneering work of Grosse and Wulkenhaar, noncommutative $\phi_{4}^{\star 4}$ theory has been shown renormalizable on four dimensional flat noncommutative Moyal space. The first renormalization proof [33] was based on the matrix representation of the Moyal product. It relies on adding to the usual propagator a marginal harmonic potential, as required by Langmann-Szabo duality [43], a process nicknamed "vulcanization". An impressive explicit computation of this propagator in the matrix base was then combined with an extensive analysis of all possible contractions of ribbon graphs in the RG equations [32]. These founding papers opened the subject of renormalizable non commutative field theories, hereafter called RNCQFT.

The initial renormalization proof was completed and improved by introducing multi-scale analysis, first in the matrix base [54], then in position space [35]. The $\beta$-function was computed at one loop in [31], then shown to vanish at all orders [23], [22] at the self-duality point $\Omega=1$ (where $\Omega$ is the coefficient of the harmonic term). The exciting conclusion is that the $\phi_{4}^{\star 4}$-theory is asymptotically safe, hence free of any Landau ghost. Wave function renormalization exactly compensates the renormalization of the four-point function, so that the flow between the bare and the renormalized coupling is bounded.

Therefore the full non-perturbative construction of the $\phi_{4}^{\star 4}$-theory should be possible. Standard constructive tools, such as cluster and Mayer expansions do not apply due to non-locality, but this problem can be overcome with a new expansion called the loop-vertex expansion [51]. This expansion also applies to the commutative case [46] 
hence provides an example where NCQFT lead to a better understanding of ordinary field theory. The full construction of $\phi_{4}^{\star 4}$-theory now requires to extend these tools to a multiscale analysis.

This is a vindication of the initial intuition of the founders of non commutative quantum field theory [55], [40], [57] that these theories should behave better than the ordinary ones in the ultraviolet regime.

Essentially most of the standard tools of field theory such as parametric [37], [53] and Mellin representations [36], dimensional regularization and renormalization [39] and the Connes-Kreimer Hopf algebra formulation of renormalization [59] have now been generalized to RNCQFT. Other RNCQFTs have been also developed such as complex models, $N$-component scalar fields, and the fermionic NC Gross-Neveu model and their flow computed in many cases [63], [62], [42], [20], [1], [2]. Their propagator can be more complicated, namely of the covariant type [38] (studied for scalar fields in [44], [45]), hence describes the influence of a constant magnetic background field. Such covariant models are important for the future applications of RNCQFT to condensed matter problems such as the quantum Hall effect [58], [49], [41].

Concerning other scalar models, in [28], [29] the noncommutative $\phi_{6}^{\star 3}$-model at the self-duality point was built and shown just renormalizable and exactly solvable. Self-duality relates this model to the Kontsevich model. For $\phi_{4}^{\star 3}$ see [27]. The $\phi_{3}^{\star 6}$ model has been shown renormalizable with $x$-space techniques in [64]. A recent review on these developments is [52].

One of the main goal for RNCQFT is to develop gauge theories in noncommutative geometry [17]. Yang-Mills theories are naturally obtained from the spectral action principle [18], [15] relative to an appropriate Dirac operator. In this way, a beautiful reformulation of the standard model of particle physics was obtained; see [16] for its most recent version.

We would like RNCQFT to connect to this reformulation, although it is also a strict extension of these ideas. Renormalizable theories survive RG flows, hence RNCQFTs might be the ones relevant to physics beyond the standard model, if noncommutativity (still confined to an "internal" space in [16]) fully invades space time itself at some still unexplored energy scale.

Therefore it is a central issue to find renormalizable noncommutative gauge theories. Usual Yang-Mills theory on Moyal space is known to be not renormalisable [47]. The principal of spectral action is to compute a one-loop effective action of Fermions in a classical external gauge field. Translated from Fermions to scalar fields as in [26], the computation was completed in position space [21] and in the matrix base [30]. The problem with such effective actions is that $A_{\mu}=0$ is not a stable solution of the classical field equation. Recently a particular non-trivial explicit vacuum was found under a radial Ansatz, as part of a more general study of a Connes-Lott model [19] (Yang-Mills theory coupled to the Higgs field) but in which the spectral action 
is derived from a non-standard Dirac operator, namely the square root of the GrosseWulkenhaar propagator. In this way the relation between the Higgs mechanism, the non-trivial gauge vacuum and the harmonic potential for the Higgs field is somewhat clarified [34].

Parallel to these developments the mathematical theory of Moyal products was generalized to a category of spaces called the solvable symmetric spaces [5]. At the origin was a question raised by A. Weinstein in the context of deformation quantization of symmetric spaces. Roughly speaking, the so-called 'WKB quantization program' for symmetric spaces may be described as follows.

An affine manifold $(M, \nabla)$ is called symmetric if the local geodesic symmetry centered at any point $x$ of $M$ globally extends as an affine transformation of $(M, \nabla)$ :

$$
s_{x}: M \rightarrow M, \quad \operatorname{Exp}_{x}^{-1} \circ s_{x} \circ \operatorname{Exp}_{x}=-\mathrm{id} .
$$

It is called a symplectic symmetric space or SSS if the symmetries preserve a nondegenerate 2-form $\omega$ (which in this case is then automatically closed). On such a space which is strictly geodesically convex (e.g., a Hermitian symmetric space of the non-compact type), one defines the following three-point function,

$$
\begin{aligned}
\Phi^{-1}: M \times M \times M & \rightarrow M \times M \times M \\
\left(x_{1}, x_{2}, x_{3}\right) & \mapsto\left(m\left(x_{1}, x_{2}\right), m\left(x_{2}, x_{3}\right), m\left(x_{3}, x_{1}\right)\right),
\end{aligned}
$$

where $m(x, y)$ is the mid-point on the geodesic line between $x$ and $y\left(s_{m(x, y)} x=y\right)$. The latter function admits an inverse $\Phi$ locally around the diagonal in $M^{3}$. Therefore, when there is no second de Rham cohomology and for 'small geodesic triangles', one may define the following local function,

$$
S_{W}:=\text { Area } \circ \Phi
$$

where, given an oriented geodesic triangle $x \stackrel{\Delta}{y} z$ in $M$, one sets

$$
\operatorname{Area}(x, y, z):=\int_{x y z} \omega .
$$

Now, given a symmetry invariant formal star product $\star$ on $(M, \omega)$ that admits the following 'WKB' form,

$$
u \star v(x)=\frac{1}{\theta^{2 n}} \int_{M \times M} A_{\theta}(x, y, z) e^{\frac{i}{\theta} S(x, y, z)} u(y) v(z) \omega_{y}^{n} \otimes \omega_{z}^{n},
$$

for some invariant (formal) amplitude $A_{\theta}$ and phase $S$. A. Weinstein suggests in [65] that the above function $S_{W}$ should be relevant in the asymptotics of the phase

$$
S \sim S_{W}
$$


The problem was thus to develop a suitable geometrical framework for the study of asymptotic quantization of symplectic symmetric spaces allowing to decide about Weinstein's asymptotics as well as to describe the amplitude $A_{\theta}$ within a non-formal context.

Two main classes have been treated in the solvable situation (i.e., when the transvection group of the symmetric space is a solvable Lie group): the class of elementary spaces [6] and the class of rank one spaces [14]. In those cases, the WKB-quantization program has been carried out explicitly and within the non-formal setting. In particular, the phase function $S$, in the solvable case, appears to coincide with Weinstein function $S_{W}$ up to a possible extra boundary term.

It is now a natural question to ask whether RNCQFT and vulcanization extend to non-trivial geometric backgrounds, as RNCQFT might eventually be applied in the presence of strong gravitational fields, e.g., in the vicinity of a black hole horizon. In order to have at least semi-infinite renormalization flows we need an infinitedimensional Moyal algebra of functions. This rules out compact spaces such as fuzzy spheres but points to the interesting case of SSS.

This paper is organized as follows:

Section 2 recalls the definitions and basic properties of the Moyal product on SSS. In Section 3 we extend the notion of Langmann-Szabo duality to SSS and compute the vulcanized Laplacian explicitly.

In Section 4 we give the action functional of the $\phi_{4}^{\star 4}$ theory on SSS. and we show that the properties of associativity and traceability of the Moyal products on SSS allow one to extend to these backgrounds the theory of Filk moves [24] and the "Moyality" of Feynman amplitudes for planar graphs with a single external face [52].

This completes essentially the model-independent steps of the renormalization program, as it generalizes to SSS the dimension-independent locality principle of ordinary geometry.

In Section 5 as a prelude to the spectral analysis of the propagator which is an essential step for renormalization, we remark that the vulcanized Laplacian kernel is a generator of a representation of the metaplectic group. This should allow access to its spectral properties, and to explicit bounds on this kernel.

In conclusion we comment briefly on the remaining steps for a full renormalization proof of vulcanized $\phi_{4}^{\star 4}$ on the simplest SSS space of dimension 4 , e.g., $\mathrm{SU}(1,2) / \mathrm{U}(2)$, which is devoted to future publications.

Acknowledgments. P. B. acknowledges partial support from the IAP grant 'NOSY' delivered by the Belgian Federal Government as well as partial support from the IISN-Belgium (convention 4.4511.06). R. G. and V. R. acknowledge the support of the ANR-Genophy grant. 


\section{Definitions and notations}

2.1. Hermitian symmetric spaces of the non-compact type. The so-called 'Moyal plane' corresponds to the deformation quantization ( $\star$-product) of the (flat) Euclidean vector space $\left(V:=\mathbb{R}^{2 n}, \beta^{0}\right)$ endowed with its canonical bilinear symplectic 2-form $\omega^{0}=\sum_{j=1}^{n} \mathrm{~d} p_{j} \wedge \mathrm{d} q_{j}$. In order to pass to a curved framework, one is, on the first hand, naturally led to consider the class of Riemannian manifolds $(M, \beta)$ endowed with a compatible symplectic structure $\omega$. By compatible, we mean that the triple $(M, \beta, \omega)$ is a Kähler manifold. On the second hand, the use of geodesic symmetries within quantization processes has been shown to be very efficient [3], [60], [65], [6]. At last, Sekigawa and Vanhecke showed in [56] that a Kähler manifold whose symplectic structure is invariant by the geodesic symmetries must be a Hermitian symmetric space, i.e., the geodesic symmetries preserve the Levi-Civita connection as well.

Another important feature of the Moyal plane is its traciality: the Hilbert space of square integrable functions $\mathscr{H}:=L^{2}\left(\mathbb{R}^{2 n}\right)$ is stable under Weyl's product $\star^{W}$ and turns out to be a Hilbert algebra in the sense that the following property holds:

$$
\int a \star^{W} b:=\int a b \quad(a, b \in \mathscr{H}) .
$$

In the curved compact situation, however, there is no hope that such a property would hold at a non-formal level [50] as shown by analyzing the simplest case of the 2 -sphere.

These considerations therefore lead us to focus on the class of Hermitian symmetric spaces of the non-compact type, which we present here only in the rank one case (the higher rank situation can be treated by applying the 'extension lemma' in [11]).

Definition 2.1. A rank one Hermitian symmetric space of the non-compact type is a homogeneous manifold $\mathrm{SU}(1, n) / \mathrm{U}(n)(n \geq 1)$.

The metric and symplectic structures may be described as follows. Denoting $G:=\mathrm{SU}(1, n)$ and $K:=\mathrm{U}(n)$, the Lie algebra $g$ of $G$ splits into a direct sum of vector spaces $\mathfrak{g}=\mathfrak{k} \oplus \mathfrak{p}$ where $\mathfrak{k}$ is the Lie algebra of the maximal compact subgroup $K$ and where $\mathfrak{p}$ is its orthonormal complement with respect to the Killing form $B$ on $\mathfrak{g}$. The above decomposition may also be seen as the $( \pm 1)$-eigenspace decomposition with respect to the Cartan involution $\sigma$ of $g$ associated with the choice of $K$. Note that in this case the Cartan involution is interior: there exists an element $j$ in the center $Z(K)=\mathrm{U}(1)$ of $K$ such that $\sigma=\operatorname{Ad}(j)$. The differential at the unit element $e$ of the projection $\pi: G \rightarrow G / K$ identifies the vector space $p$ with the tangent space $T_{K}(\mathbb{D})$ at $K$ to the manifold $\mathbb{D}:=G / K$. The vector space $\mathfrak{p}$ is an invariant subspace under the (adjoint) action of $K$ on $\mathfrak{g}$. Moreover, the tensors $\left.B\right|_{\mathfrak{p} \times \mathfrak{p}}, \mathcal{F}:=\left.\operatorname{ad}(\log \boldsymbol{j})\right|_{\mathfrak{p}}$ and $\omega:=\left.B\right|_{\mathfrak{p} \times \mathfrak{p}} \circ$ id $\otimes \mathcal{A}$ are invariant under the $K$-action. They globalize to $\mathbb{D}$ as respectively the metric $\beta$, the complex structure $J$ and the symplectic (Kähler) 
2-form $\omega$. The geodesic symmetry $s_{g K}^{\mathbb{D}}$ centered at $g K$ with respect to the LeviCivita connection $\nabla^{\mathbb{D}}$ of $\beta$ is explicitly described by the following expression:

$$
s_{g K}^{\mathbb{D}}(h K)=g \boldsymbol{j} g^{-1} h \boldsymbol{j}^{-1} K .
$$

A second picture of the geometry of $\mathbb{D}$ relies on the (co)adjoint representation. Indeed, the adjoint orbit $\mathcal{O}$ of $Z:=\log (\boldsymbol{j})$ turns out to be isomorphic as a $G$-homogeneous space to $\mathbb{D}$. The symplectic structure identifies with the KKS-form:

$$
\omega_{x}\left(X^{\star}, Y^{\star}\right)=B(x,[X, Y])
$$

where $x \in \mathcal{O} \subset \mathfrak{g}$ and where $X^{\star}$ denotes the fundamental vector field $X_{x}^{\star}:=$ $\left.\frac{d}{d t}\right|_{0} \operatorname{Ad}(\exp (-t X)) x$ associated to $X \in \mathfrak{g}$. The metric is then simply the induced metric on $\mathcal{O}$ from the Killing form $B$ on $g$.

A third and last picture is based on the Iwasawa decomposition of $G$. Recall that all Abelian subalgebras of $g$ contained in $\mathfrak{p}$ are $K$-conjugated. Let $a \subset \mathfrak{p}$ be such an Abelian subalgebra. Its adjoint action is then semisimple and on gets a decomposition of $g$ into root spaces:

$$
\mathfrak{g}=\mathfrak{g}_{0} \oplus \bigoplus_{\alpha \in \Phi} \mathfrak{g}_{\alpha}
$$

where for $\alpha \in a^{\star}$, one sets $\mathfrak{g}_{\alpha}:=\{X \in \mathfrak{g} \mid[H, X]=\alpha(H) X, H \in \mathfrak{a}\}$ and where $\Phi$ is the subset of $a^{\star} \backslash 0$ for which $g_{\alpha}$ is not reduced to zero. A choice of an orthonormal basis of a yields a partition of the set $\Phi$ into $\Phi^{+} \cup \Phi^{-}$with $\Phi^{-}=-\Phi^{+}$such that the subspace

$$
\mathfrak{n}:=\bigoplus_{\alpha \in \Phi^{+}} g_{\alpha}
$$

is a (nilpotent) subalgebra of $\mathrm{g}$. In the rank one case with $n \geq 2$, one has $\operatorname{dim} a=1$, $\Phi^{+}=\{\alpha, 2 \alpha\}, \operatorname{dim} g_{2 \alpha}=1$ and $\mathfrak{n}$ is isomorphic to a Heisenberg algebra. ${ }^{1}$ The direct sum $\mathfrak{s}=a \oplus \mathfrak{n}$ is then a solvable subalgebra of $\mathfrak{g}$ whose associated simply connected Lie group $\mathbb{S}$ is an exponential $H$-group (one dimensional split extension of a Heisenberg group). The map

$$
\mathfrak{s}=a \oplus \mathfrak{g}_{\alpha} \oplus \mathfrak{g}_{2 \alpha} \longrightarrow \mathbb{D}:(a, x, z) \mapsto \exp (a) \exp (x) \exp (z) K
$$

turns out to be a global Darboux diffeomorphism when $\mathfrak{s}$ is endowed with the bilinear form $\omega=\mathrm{d} a \wedge \mathrm{d} z+\omega^{0}$ where $\omega^{0}$ denotes the symplectic form on $g_{\alpha}$ inducing the bracket in the Heisenberg algebra $n$. Note that the above map define global coordinates on the group $\mathbb{S}$ on which the 2 -form $\omega$ is left-invariant. The identifications $\mathbb{S}=\mathcal{O}=\mathbb{D}$ are then $\$$-equivariant symplectic identifications.

\footnotetext{
${ }^{1}$ Note that $\alpha$ can be normalized to 1 .
} 
2.2. Contracted spaces. The symplectic Lie group $\mathbb{S}$ is 'of symmetric type' in the sense that it carries a symmetric affine connection $\left(\nabla^{\mathbb{D}}\right)$ which is left-invariant. However, there exists on $\mathbb{S}$ another symmetric affine connection, $\nabla^{c}$, left-invariant and moreover symplectic (i.e., $\nabla^{c} \omega=0$ ). This connection is curved and is not the LeviCivita connection associated to any Riemannian or pseudo-Riemannian metric. It is a purely symplectic connection. Below we describe some features of its associated geometry. One defines the smooth map $s: \mathbb{S} \times \mathbb{S} \rightarrow \mathbb{S}:(x, y) \mapsto s_{x}(y)$ given in coordinates $(a, x, y)$ by

$$
\begin{aligned}
& s_{(a, x, z)}\left(a^{\prime}, x^{\prime}, z^{\prime}\right)=\left(2 a-a^{\prime}, 2 \cosh \left(a-a^{\prime}\right) x-x^{\prime},\right. \\
&\left.2 \cosh \left(2\left(a-a^{\prime}\right)\right) z+\omega^{0}\left(x, x^{\prime}\right) \sinh \left(a-a^{\prime}\right)-z^{\prime}\right) .
\end{aligned}
$$

One then observes that it is $\$$-equivariant (i.e., $x s_{y} x^{-1} z=s_{x y} z$ for all $x, y, z \in$ $\$)$. Moreover for every $x$, the map $s_{x}$ is an involutive symplectomorphism of $(\mathbb{\$}, \omega)$. At last, one has the identity: $s_{x} s_{y} s_{x}=s_{s_{x}}(y)$. The following formula defines an affine connection on $\mathbb{\$}$,

$$
\omega_{x}\left(\nabla_{X}^{c} Y, Z\right):=\frac{1}{2} X_{x} \cdot \omega\left(Y+s_{x_{\star}} Y, Z\right),
$$

where $X, Y, Z$ are any vector fields on $\mathbb{\$}$. The connection $\nabla^{c}$ turns out to be torsion-free, symplectic, and is the only affine connection which is invariant under the involutions $\left\{s_{x}\right\}_{x \in \Phi}$. The latter involutions actually coincide with the geodesic symmetries associated to $\nabla^{c}$, i.e., the triple $(\mathbb{S}, \omega, s)$ is a symplectic symmetric space ([5], [10]). We call it the contracted space. Its transvection group $G^{c}$ generated by even products of symmetries is a transitive solvable Lie group of affine symplectomorphisms of $(\$, \omega, s)$. It plays the role of $G$ in the non-contracted situation of $\mathbb{D}$. Similarly as $G$, the group $G^{c}$ contains $\$$ as a subgroup.

\subsection{WKB-quantization}

Theorem 2.1. For all non-zero $\theta \in \mathbb{R}$, there exists a Fréchet function space $\mathcal{E}_{\theta}$,

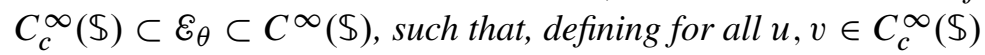

$$
\begin{aligned}
\left(u \star_{\theta} v\right)\left(a_{0}, x_{0}, z_{0}\right) \\
=\frac{1}{\theta^{\operatorname{dim} \Phi}} \int_{\$ \times \Phi} \cosh \left(2\left(a_{1}-a_{2}\right)\right)\left[\cosh \left(a_{2}-a_{0}\right) \cosh \left(a_{0}-a_{1}\right)\right]^{\operatorname{dim} \Phi-2} \\
\quad \times \exp \left(\frac { 2 i } { \theta } \left\{S^{0}\left(\cosh \left(a_{1}-a_{2}\right) x_{0}, \cosh \left(a_{2}-a_{0}\right) x_{1}, \cosh \left(a_{0}-a_{1}\right) x_{2}\right)\right.\right. \\
\left.\left.\quad-\circlearrowright \sinh \left(2\left(a_{0}-a_{1}\right)\right) z_{2}\right\}\right) u\left(a_{1}, x_{1}, z_{1}\right) v\left(a_{2}, x_{2}, z_{2}\right) d a_{1} d a_{2} d x_{1} d x_{2} d z_{1} d z_{2}
\end{aligned}
$$

where $S^{0}\left(x_{0}, x_{1}, x_{2}\right):=\omega^{0}\left(x_{0}, x_{1}\right)+\omega^{0}\left(x_{1}, x_{2}\right)+\omega^{0}\left(x_{2}, x_{0}\right)$ is the phase for the

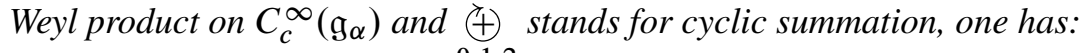


(1) $u \star_{\theta} v$ is smooth and the map $C_{c}^{\infty}(\mathbb{\$}) \times C_{c}^{\infty}(\$) \rightarrow C^{\infty}(\$)$ extends to an associative product on $\mathcal{E}_{\theta}$. The pair $\left(\mathcal{E}_{\theta}, \star_{\theta}\right)$ is a $\left(\right.$ pre- $\left.C^{\star}\right)$ Fréchet algebra.

(2) In coordinates $(a, x, z)$ the group multiplication law reads

$$
L_{(a, x, z)}\left(a^{\prime}, x^{\prime}, z^{\prime}\right)=\left(a+a^{\prime}, e^{-a^{\prime}} x+x^{\prime}, e^{-2 a^{\prime}} z+z^{\prime}+\frac{1}{2} \omega^{0}\left(x, x^{\prime}\right) e^{-a^{\prime}}\right) .
$$

The phase and amplitude occurring in formula (5) are both invariant under the left action $L: \mathbb{\$} \times \mathbb{\$} \rightarrow \mathbb{\$}$.

(3) Formula (5) admits a formal asymptotic expansion of the form

$$
u \star_{\theta} v \sim u v+\frac{\theta}{2 i}\{u, v\}+O\left(\theta^{2}\right),
$$

where $\{$,$\} denotes the symplectic Poisson bracket on C^{\infty}(\$)$ associated with $\omega$. The full series yields an associative formal star product on $(\$, \omega)$ denoted by $\tilde{\star}_{\theta}$.

Roughly speaking, the above product can be obtained by intertwining the standard Weyl-Moyal product $\star_{\theta}^{0}$ on the Schwartz space $\delta(\mathfrak{s})=: \&$ (endowed with the Poisson structure associated with the 2-form $\omega$ ). Indeed, one formally has

$$
u \star_{\theta} v=T_{\theta, 1}\left(T_{\theta, 1}^{-1} u \star_{\theta}^{0} T_{\theta, 1}^{-1} v\right),
$$

where, denoting by

$$
\mathcal{F}_{3} u(a, v, \xi):=: \hat{u}(a, v, \xi):=\int e^{-i \xi z} u(a, v, \ell) \mathrm{d} \ell
$$

the partial Fourier transform in the $z$-variable and defining the following smooth one-parameter family of diffeomorphisms

$$
\phi_{\theta}:(a, v, \xi) \mapsto\left(a, \frac{1}{\cosh (\theta \xi)} v, \frac{1}{2 \theta} \sinh (2 \theta \xi)\right),
$$

the $T_{\theta, 1}$ map is defined as

$$
T_{\theta, 1}:={\mathcal{F}_{3}}^{-1} \circ\left(\phi_{\theta}^{-1}\right)^{\star} \circ \mathscr{F}_{3}: \& \rightarrow \delta^{\prime} .
$$

The space $\varepsilon_{\theta}$ then corresponds to the range of the above map in the tempered distributions $\ell^{\prime}$.

In dimension two $(n=1)$, the space $\mathrm{SU}(1,1) / \mathrm{U}(1)$ is isometric to the hyperbolic plane. The algebra $\mathfrak{n}$ is then one dimensional ( $g_{\alpha}$ disappears) and the group $\mathbb{S}$ is isomorphic to the affine group $a x+b$ whose group law, in coordinates $(a, z)$, reads $(a, z) \cdot\left(a^{\prime}, z^{\prime}\right)=\left(a+a^{\prime}, e^{-2 a^{\prime}} z+z^{\prime}\right)$. The product formula (5) degenerates as

$$
\begin{gathered}
\left(u \star_{\theta} v\right)\left(a_{0}, z_{0}\right)=\frac{1}{\theta^{2}} \int_{\Phi \times \Phi} \cosh \left(2\left(a_{1}-a_{2}\right)\right) \exp \left(\frac{-2 i}{\theta} \underset{0,1,2}{\uplus} \sinh \left(2\left(a_{0}-a_{1}\right)\right) z_{2}\right) \\
\cdot u\left(a_{1}, z_{1}\right) v\left(a_{2}, z_{2}\right) d a_{1} d a_{2} d z_{1} d z_{2} .
\end{gathered}
$$

Again it can be seen as transporting Weyl-Moyal's product by a intertwiner of the above form (7) with $\phi_{\theta}(a, \xi):=\left(a, \frac{1}{2 \theta} \sinh (2 \theta \xi)\right)$. 
2.4. Traciality. The above products are not tracial in the sense that $L^{2}(\mathbb{\$})$ is stable and that property (2) holds. Nevertheless, they can be easily modified into strongly tracial products [8].

Proposition 2.1. Let $\theta>0$ and denote by $L^{2}(\$)$ the space of functions on $\$$ that are square integrable with respect to a left-invariant Haar measure. Set

$$
S:=S^{0}\left(\cosh \left(a_{1}-a_{2}\right) x_{0}, \cosh \left(a_{2}-a_{0}\right) x_{1}, \cosh \left(a_{0}-a_{1}\right) x_{2}\right)-\underset{0,1,2}{\leftrightarrows} \sinh \left(2\left(a_{0}-a_{1}\right)\right) z_{2}
$$

for the phase function as in the product formula (5). Let $u, v \in C_{c}^{\infty}(\mathbb{\$})$. Then the formula

$$
u \star_{\theta}^{\mathrm{can}} v=\frac{1}{\theta^{\operatorname{dim} \Phi}} \int_{\Phi \times \Phi} \boldsymbol{A}_{\mathrm{can}} e^{\frac{2 i}{\theta} S} u \otimes v
$$

where

$$
\begin{aligned}
\boldsymbol{A}_{\mathrm{can}}\left(x_{0}, x_{1}, x_{2}\right):= & \sqrt{\cosh \left(2\left(a_{0}-a_{1}\right)\right) \cosh \left(2\left(a_{1}-a_{2}\right)\right) \cosh \left(2\left(a_{2}-a_{0}\right)\right)} \\
& \cdot\left[\cosh \left(a_{0}-a_{1}\right) \cosh \left(a_{1}-a_{2}\right) \cosh \left(a_{2}-a_{0}\right)\right]^{\frac{\operatorname{dim} \$-2}{2}},
\end{aligned}
$$

extends to a complex bilinear associative product $\star_{\theta}^{\mathrm{can}}: L^{2}(\mathbb{\$}) \times L^{2}(\mathbb{\$}) \rightarrow L^{2}(\mathbb{\$})$. The pair $\left(L^{2}(\$), \star_{\theta}^{c a n}\right)$ is a Hilbert algebra on which the symmetries $\left\{s_{x}\right\}_{x \in \$}$ act by isometries. Moreover, the traciality property (2) holds.

In dimension two, one has ([12], [6])

Proposition 2.2. Let $\theta>0$ and denote by $L^{2}(\$)$ the space of functions on $S$ that are square integrable with respect to a left-invariant Haar measure. Let $u, v \in C_{c}^{\infty}(\mathbb{S})$ $(n=1)$. Then the formula

$$
\begin{aligned}
& \left(u \star_{\theta}^{\mathrm{can}} v\right)\left(a_{0}, z_{0}\right) \\
& =\frac{1}{\theta^{2}} \int_{\Phi \times \$}\left[\cosh \left(2\left(a_{0}-a_{1}\right)\right) \cosh \left(2\left(a_{1}-a_{2}\right)\right) \cosh \left(2\left(a_{2}-a_{0}\right)\right)\right]^{\frac{1}{2}} \\
& \quad \cdot \exp \left(\frac{-2 i}{\theta} \underset{0,1,2}{\leftrightarrows} \sinh \left(2\left(a_{0}-a_{1}\right)\right) z_{2}\right) u\left(a_{1}, z_{1}\right) v\left(a_{2}, z_{2}\right) d a_{1} d a_{2} d z_{1} d z_{2}
\end{aligned}
$$

extends to a complex bilinear associative product $\star_{\theta}^{\mathrm{can}}: L^{2}(\$) \times L^{2}(\$) \rightarrow L^{2}(\$)$. The pair $\left(L^{2}(\$), \star_{\theta}^{c a n}\right)$ is a Hilbert algebra on which the symmetries $\left\{s_{x}\right\}_{x \in \Phi}$ act by isometries. Moreover, the traciality property (2) holds.

These tracial products may be seen as transporting Weyl-Moyal by a modified intertwiner of the form

$$
T_{\theta, \text { can }}:={\mathcal{F}_{z}}_{z}^{-1} \circ \mathcal{M} \sqrt{\mathrm{Jac}_{\phi_{\theta}^{-1}}} \circ\left(\phi^{-1}\right)_{\theta}^{\star} \circ \mathcal{F}_{z},
$$


where $\mathcal{M}_{f}$ denotes the multiplication operator by $f$. The above map actually defines a unitary automorphism

$$
T_{\theta, \text { can }}: L^{2}(\$) \longrightarrow L^{2}(\$),
$$

where we identified $L^{2}(\$)$ with $L^{2}(\mathfrak{s})$ via the global Darboux chart (3).

\section{LS-duality}

3.1. Definition. Within the flat context, Langmann-Szabo duality coincides with the symplectic Fourier transform. It plays a key role in making the corresponding field theory renormalizable (perturbatively and probably also at the constructive level, see the introduction).

On curved Hermitian symmetric spaces we have natural notions of Laplacian and of invariant star product but LS duality has to be defined. In this section we make a first proposal in this direction: simply transport the ordinary LS duality in the flat case to the curved case under the $T$ transformation. Then we consider the curved Laplacian (which is certainly not spectrally equivalent to the flat one) and extend it into an LS covariant operator by adding $\Omega^{2}$ times its LS dual. This procedure mimics the Grosse-Wulkenhaar one but does not lead to an equivalent theory simply because when the Grosse-Wulkenhaar $\Omega$ parameter is turned off, the limits are different.

More precisely, on the space $\delta$ of Schwartz functions defined on the symplectic vector space $\left(\mathfrak{s}, \omega:=\mathrm{d} a \wedge \mathrm{d} z+\omega^{0}\right)$, one defines the following integral transformations:

$$
\widetilde{F}_{ \pm \omega}(u)(x):=\int_{\mathfrak{s}} e^{\mp i \omega(x, y)} u(y) \mathrm{d} y .
$$

These then define topological automorphisms of $\delta$ extending as unitary isomorphisms to $L^{2}(\mathfrak{s})$.

Definition 3.1. The $( \pm) L S$-transform on $L^{2}(\$)$ is the involutive unitary automorphism defined as

$$
\widetilde{F}_{\text {can }}^{ \pm}:=T_{\theta, \text { can }} \circ \mathscr{F}_{ \pm \omega} \circ T_{\theta \text {,can }}^{-1} .
$$

Our intertwiner may be expressed in terms of the flat LS-transform, indeed the following lemma holds by a straightforward computation.

Lemma 3.1. Denote by $\Phi_{\theta}: \$ \rightarrow \$$ the following one parameter family of diffeomorphisms:

$$
\Phi_{\theta}(a, v, z):=\left(\frac{1}{2 \theta} \sinh (2 \theta a), \cosh (\theta a) v, z\right) .
$$

Then one has

$$
\left.T_{\theta, 1}^{-1}=\mathscr{F}_{\omega} \circ \mathcal{M}_{[\cosh (\theta a)]}\right]^{\operatorname{dim} \Phi-2} \circ \Phi_{\theta}^{\star} \circ \mathcal{F}_{\omega} .
$$


3.2. The Laplacian. We first describe the standard Laplace operator on $G / K$ identified with $\mathbb{\$}$. As we have seen, the Laplace operator $\Delta$ is a second order left-invariant differential operator on the Lie group $\mathbb{\$}$. Moreover its principal symbol coincides with the quadratic form induced by the invariant metric $\beta$ on $G / K$. It is therefore of the form $B_{i j} \widetilde{X}_{i} \widetilde{X}_{j}+B_{i} \tilde{X}_{i}+B$, where $\left\{X_{i}\right\}$ is a basis of $\mathfrak{s}$ and where $B_{i j}=B\left(X_{i \mathfrak{p}}, X_{j \mathfrak{p}}\right)$. Note that for all $X, Y \in \mathfrak{g}$, since $Z$ is central in $\mathfrak{f}$ and orthogonal to $\mathfrak{p}$, the invariance of the Killing form yields: $\omega\left(X_{\mathfrak{p}}, Y_{\mathfrak{p}}\right)=B(Z,[X, Y])$. In the same way, one gets $B\left(X_{\mathfrak{p}}, Y_{\mathfrak{p}}\right)=B(Z,[[Z, X], Y])$. Observe also that $X_{\mathfrak{p}}=-[Z,[Z, X]]$. Now $B\left(H, \mathfrak{n}_{\mathfrak{p}}\right)=B(Z,[[Z, H], \mathfrak{n}])=B([Z,[Z, H]], \mathfrak{n})=B(H, \mathfrak{n})=0$. Similarly, $B\left(\mathfrak{g}_{2 \alpha_{\mathfrak{p}}}, \mathfrak{g}_{\alpha_{\mathfrak{p}}}\right)=B\left(Z,\left[\left[Z, \mathfrak{g}_{2 \alpha}\right], \mathfrak{g}_{\alpha_{\mathfrak{p}}}\right]\right)=B\left(\mathfrak{g}_{2 \alpha_{\mathfrak{p}}}\right)=B\left(\mathfrak{g}_{2 \alpha}-\sigma \mathfrak{g}_{2 \alpha}, \mathfrak{g}_{\alpha}\right)=$ $B\left(\mathfrak{g}_{2 \alpha}+\mathfrak{g}_{-2 \alpha}, \mathfrak{g}_{\alpha}\right)=0$. In particular, since $a \oplus \mathfrak{g}_{2 \alpha}$ and $\mathfrak{g}_{\alpha}$ are symplectic, there are also stable under the complex structure $\mathcal{L}$. Fix then a Lagrangian subspace $\mathscr{L}$ of $\mathfrak{g}_{2 \alpha}$ in duality with $\mathscr{L}^{\prime}:=\mathscr{L}(\mathscr{L})$. Consider an orthonormal basis $\left\{e_{k}\right\}$ of $\mathscr{L}$ as well as its dual basis $\left\{f_{k}:=\mathscr{L}\left(e_{k}\right)\right\}$ in $\mathscr{L}^{\prime}$. Observe that the left-invariant vector fields are expressed as:

$$
\begin{aligned}
\tilde{H} & =\left.\frac{d}{d t}\right|_{0} \exp (a H) \exp (x) \exp (z E) \exp (t H) \\
& =\left.\frac{d}{d t}\right|_{0} \exp ((a+t) H) \exp \left(e^{-t} x\right) \exp \left(z e^{-2 t} E\right)=\partial_{a}-x^{j} \partial_{x^{j}}-2 z \partial_{z}, \\
\tilde{v} & =\left.\frac{d}{d t}\right|_{0} \exp (a H) \exp (x+t v) \exp \left(\left(z+\frac{1}{2} t \omega(x, v)\right) E\right)=\partial_{v}+\frac{1}{2} \omega(x, v) \partial_{z}, \\
\tilde{E} & =\partial_{z} .
\end{aligned}
$$

In particular, the fields $\tilde{v}$ as well as $\widetilde{E}$ preserve the Liouville volume. By imposing self-adjointness, one observes that the Laplace operator admits the expression

$$
\Delta=\tilde{H}^{2}+\sum_{k}\left(\tilde{e}_{k}^{2}+\tilde{f}_{k}^{2}\right)+\tilde{E}^{2}-(\operatorname{dim} \$) \tilde{H},
$$

where we adopt the normalization $\omega(H, E)=2 B(Z, E):=1$.

For instance, in the $n=2$ case, one has the following matrix representation:

$$
\begin{gathered}
\sigma A=-A^{\dagger}, \quad \mathfrak{k}=\left\{\left(\begin{array}{cc}
-\operatorname{tr} X & 0 \\
0 & X
\end{array}\right) \mid X \in \mathfrak{u}(2)\right\}, \quad \mathfrak{p}=\left\{\left(\begin{array}{ll}
0 & v^{\dagger} \\
v & 0
\end{array}\right) \mid v \in \mathbb{C}^{2}\right\}, \\
Z=\frac{1}{3}\left(\begin{array}{cc}
-2 i & 0 \\
0 & i I
\end{array}\right), \quad H=\left(\begin{array}{lll}
0 & 1 & 0 \\
1 & 0 & 0 \\
0 & 0 & 0
\end{array}\right), \quad E=\left(\begin{array}{ccc}
i & -i & 0 \\
i & -i & 0 \\
0 & 0 & 0
\end{array}\right), \\
\xi=\left(\begin{array}{ccc}
0 & 0 & \bar{\xi} \\
0 & 0 & \bar{\xi} \\
\xi & -\xi & 0
\end{array}\right), \quad E_{\mathfrak{p}}=\left(\begin{array}{ccc}
0 & -i & 0 \\
i & 0 & 0 \\
0 & 0 & 0
\end{array}\right), \quad \xi_{\mathfrak{p}}=\left(\begin{array}{lll}
0 & 0 & \bar{\xi} \\
0 & 0 & 0 \\
\xi & 0 & 0
\end{array}\right),
\end{gathered}
$$

where $\xi:=: x e_{1}+y f_{1}=: x+i y$ and ${ }^{\dagger}$ stands for transposed conjugated.

In order to define a propagator that is invariant under the LS-transform, we first recall some facts. View the space $\mathbb{S}$ as the adjoint orbit $\mathcal{O}$ in $g$ endowed with the KKS 
form $\omega$ as described in Section 2.1. Consider $(\mathcal{O}, \omega, G)$ as a strongly Hamiltonian system and denote by

$$
\lambda: \mathfrak{g} \rightarrow C^{\infty}(\mathcal{O}): X \mapsto \lambda_{X}
$$

the associated classical moment mapping (i.e., $\lambda_{X}(x):=B(x, X)$ ). Express the latter within Iwasawa coordinates (3) as $\lambda_{X}(a, v, z):=\lambda_{X}(\exp (a H) \exp (v+z E) Z)$. One then has

Proposition 3.1 ([13]). The Moyal formal star product $\star_{\nu}^{0}$ on $\left(\mathfrak{s}, \omega:=\mathrm{d} a \wedge \mathrm{d} z+\omega^{0}\right)$ is $\mathfrak{s - c o v a r i a n t ~ i n ~ t h e ~ s e n s e ~ t h a t ~ f o r ~ a l l ~} X, Y \in \mathfrak{s}$, one has

$$
\left[\lambda_{X}, \lambda_{Y}\right]_{\star_{v}^{0}}=2 v \lambda_{[X, Y]} .
$$

At the formal level, this yields a representation of $\mathfrak{s}$ acting on $\mathbb{E}_{v}:=C^{\infty}(\mathbb{\$})[[v]]$ by derivations of the Moyal product

$$
\rho_{\nu}: \mathfrak{s} \rightarrow \operatorname{Der}\left(\mathbb{E}_{v}\right): \rho_{\nu}(X) u:=\frac{1}{2 v}\left[\lambda_{X}, u\right]_{\star_{v}^{0}} .
$$

From [13], one finds the following expressions:

$$
\begin{aligned}
\rho_{v}(E) u & =\frac{-1}{v} e^{-2 a} \sinh \left(2 v \partial_{z}\right) u, \\
\rho_{v}(w) u & =e^{-a}\left(\cosh \left(v \partial_{z}\right) \partial_{w} u-\frac{1}{v} \omega(v, w) \sinh \left(v \partial_{z}\right) u\right), \\
\rho_{v}(H) u & =-\partial_{a} u .
\end{aligned}
$$

One then has

Proposition 3.2. Let $X \in \mathfrak{s}$ and denote by $X^{\star}$ the associated right-invariant vector field on the group $\mathbb{\$}$. Then

$$
T_{\theta, \mathrm{can}} \circ \rho_{\nu}(X) \circ T_{\theta, \mathrm{can}}^{-1}=X^{\star} .
$$

Proof. We have seen that $T_{\theta \text {, an }}=\mathscr{\ell} \circ T_{\theta, 1} \circ \mathcal{C}^{-1}$ where $\mathcal{C}:=\mathscr{F}_{3}^{-1} \mathcal{M}_{P} \mathcal{F}_{3}$ for some complex-valued one variable function $P$. Proposition 2.2 of [13] tells us that $T_{\theta \text {, can }} \circ \rho_{\nu}(X) \circ T_{\theta \text {, can }}^{-1}=\mathscr{C} \circ X^{\star} \circ \mathcal{C}^{-1}$. Observing that the operator $\mathcal{C}$ is nothing else than a left-invariant convolution operator on the Lie group $\mathbb{\$}$ [7] and since $X^{\star}$ is the infinitesimal generator of the left multiplication on $\mathbb{\$}$, one obtains the result.

Chirality. The scalar Laplacian operator on $\mathbb{D}=\mathbb{\$}$ is a left-invariant differential operator. It therefore may be expressed in terms of a basis $\left\{X_{j}\right\}$ of $\mathfrak{s}$ as $\Delta^{L}=\beta_{j k} \tilde{X}_{j} \tilde{X}_{k}+\beta_{j} \tilde{X}_{j}+\beta$, where $\beta_{j k}, \beta_{j}$ and $\beta$ are real constants and where $\tilde{X}$ denotes the left-invariant vector field on $\mathbb{\$}$ associated to $X \in \mathfrak{s}$.

In the same way, one has a right-invariant operator $\Delta^{R}$ expressed in terms of the right-invariant vector fields $X_{j}^{\star}$ associated with a right-invariant Riemannian metric on $\$$. Of course, intrinsically, the left and right-geometries on $\mathbb{S}$ are isometric, essentially under the inversion map. 
Conventionally, we choose to consider the right-invariant Laplacian together with the left-invariant star products on $\mathbb{\$}$. Proposition 3.2 then yields the following expression for $\Delta^{R}$ :

$$
\Delta^{R} u=\frac{1}{4 v^{2}} \beta_{j k}\left[\left[\Lambda_{X_{j}}\left[\Lambda_{X_{k}}, u\right]_{\star_{v}}\right]_{\star_{v}}+\frac{1}{2 v} \beta_{j}\left[\Lambda_{X_{j}}, u\right]_{\star_{v}}+\beta u .\right.
$$

Here $\star_{v}$ denotes the $\$$-invariant formal star product intertwined with $\star_{v}^{0}$ under (the formal asymptotic expansion of) $T_{\theta \text {,can }}$ and $\Lambda$ denotes the associated intertwined quantum moment (see [9] for details about the latter asymptotic expansion).

3.3. LS dual of the Laplacian. In view of LS-invariance, one is therefore left with considering the following formal operator for every $X$ in $\mathfrak{s}$ :

$$
u \mapsto \mathcal{F}_{\text {can }}^{ \pm} \circ T_{\theta, \text { can }} \circ \rho_{\nu}(X) \circ T_{\theta, \text { can }}^{-1} \circ \mathcal{F}_{\text {can }}^{ \pm}(u) .
$$

Equivalently:

$$
\mathcal{F}_{\text {can }}^{ \pm} \circ T_{\theta, \text { can }} \circ \rho_{\nu}(X) \circ T_{\theta \text {,can }}^{-1} \circ \mathcal{F}_{\text {can }}^{ \pm}=T_{\theta \text {,can }} \circ \mathcal{F}_{+\omega} \circ \rho_{\nu}(X) \circ \mathcal{F}_{+\omega} \circ T_{\theta \text {,can }}^{-1} .
$$

A direct computation then yields

Lemma 3.2. With $v=\frac{\theta}{i}$, one has:

$$
\begin{aligned}
\mathcal{F}_{+\omega} \rho_{v}(H) \mathcal{F}_{+\omega} u(a, v, z) & =i z u(a, v, z), \\
\mathcal{F}_{+\omega} \rho_{v}(w) \mathcal{F}_{+\omega} u(a, v, z) & =e^{i \partial_{z}}\left[i \cosh (\theta a) \omega(v, w)-\frac{1}{\theta} \sinh (\theta a) \partial_{w}\right] u(a, v, z), \\
\mathcal{F}_{+\omega} \rho_{v}(E) \mathcal{F}_{+\omega} u(a, v, z) & =-\frac{i}{\theta} \sinh (2 \theta a) e^{2 i \partial_{z}} u(a, v, z) .
\end{aligned}
$$

Intertwining the latter by the (partial) Fourier transform (6), we obtain the following expressions:

$$
\begin{aligned}
& \mathcal{F}_{3} \mathcal{F}_{+\omega} \rho_{v}(H) \mathcal{F}_{+\omega} u(a, v, \xi)=-\partial_{\xi} \hat{u}(a, v, \xi), \\
& \mathcal{F}_{3} \mathcal{F}_{+\omega} \rho_{v}(w) \mathcal{F}_{+\omega} u(a, v, \xi)=e^{-\xi}\left[i \cosh (\theta a) \omega(v, w)-\frac{1}{\theta} \sinh (\theta a) \partial_{w}\right] \hat{u}(a, v, \xi), \\
& \mathcal{F}_{3} \mathcal{F}_{+\omega} \rho_{v}(E) \mathcal{F}_{+\omega} u(a, v, \xi)=-\frac{i}{\theta} \sinh (2 \theta a) e^{-2 \xi} \hat{u}(a, v, \xi) .
\end{aligned}
$$

Also,

$$
\begin{aligned}
\mathscr{F}_{3} \rho_{v}(w) u & =e^{-a}\left[\cosh (\theta \xi) \partial_{w} \hat{u}+\frac{i}{\theta} \omega(v, w) \sinh (\theta \xi)\right] \hat{u}, \\
\mathcal{F}_{3} \rho_{v}(H) u & =-\partial_{a} \hat{u}, \\
\mathcal{F}_{3} \rho_{\nu}(E) u & =-\frac{i}{\theta} \sinh (2 \theta \xi) e^{-2 a} \hat{u} .
\end{aligned}
$$

Noting the perfect symmetry in variables $a$ and $\xi$ between these expressions, we choose to work at the level of the space $\widetilde{\mathbb{S}}:=\{(a, u, \xi)\}$. 
Proposition 3.3. Set

$$
\mathcal{T}:=\mathcal{F}_{z}^{-1} \circ \mathcal{M} \sqrt{\operatorname{Jac}_{\phi_{\theta}^{-1}}} \circ\left(\phi^{-1}\right)_{\theta}^{\star}
$$

Then, for all $\Omega \in \mathbb{R}$, one has

$$
\begin{aligned}
\Delta^{L S}= & \mathcal{T}^{-1}\left(\Omega^{2} \mathcal{F}_{\text {can }}^{ \pm} \Delta^{R} \mathcal{F}_{\text {can }}^{ \pm}+\Delta^{R}\right) \mathcal{T} \\
= & \Omega^{2} \partial_{\xi}^{2}+\partial_{a}^{2}+\left(\frac{\Omega^{2}}{\theta^{2}} e^{-2 \xi} \sinh ^{2}(\theta a)+e^{-2 a} \cosh ^{2}(\theta \xi)\right) \Delta_{V} \\
& -\frac{2 i}{\theta}\left(\Omega^{2} e^{-\xi} \sinh 2 \theta a-e^{-a} \sinh 2 \theta \xi\right) \mathfrak{F}_{V}+\operatorname{dim}(\mathbb{S})\left(\Omega^{2} \partial_{\xi}+\partial_{a}\right) \\
& -\frac{1}{\theta^{2}}\left(\Omega^{2} e^{-4 \xi} \sinh ^{2}(2 \theta a)+e^{-4 a} \sinh ^{2}(2 \theta \xi)\right) \\
& -\left(\Omega^{2} e^{-2 \xi} \cosh ^{2}(\theta a)+e^{-2 a} \frac{1}{\theta^{2}} \sinh ^{2}(\theta \xi)\right)|v|^{2},
\end{aligned}
$$

where $\mathfrak{5}_{V}$ denotes the Euler vector field on $V$,

$$
\mathfrak{S}_{V}:=\sum_{k}\left(\omega\left(v, e_{k}\right) \partial_{e_{k}}+\omega\left(v, f_{k}\right) \partial_{f_{k}}\right),
$$

$\Delta_{V}$ denotes the Laplacian on $V$,

$$
\Delta_{V}:=\sum_{k}\left(\partial_{e_{k}}^{2}+\partial_{f_{k}}^{2}\right)
$$

and

$$
|v|^{2}:=\sum_{k}\left(\omega^{2}\left(v, e_{k}\right)+\omega^{2}\left(v, f_{k}\right)\right) .
$$

\section{Scalar field theory on SSS}

In this section we give first the general form of a scalar field theory on SSS space with polynomial action, then derive a Moyality principle for that action (at leading order), assuming that the propagator at high energy behaves like the flat space one, which is a reasonable assumption.

4.1. The action. The quantum field theory of a scalar in a symmetric space is defined by the generic action

$$
S=\int \phi \Delta^{L S} \phi+V(\phi)_{\star}
$$

where $V$ is some $\star$ polynomial in $\phi$ and $\Delta_{L S}$ is the vulcanized Laplacian defined in (10). 
The flat Fourier transform can be written as

$$
\mathcal{F}_{+\omega}(f)=\delta \star_{\theta}^{0} f, \quad \mathcal{F}_{-\omega}(f)=f \star_{\theta}^{0} \delta .
$$

with $\delta \star_{\theta}^{0} \delta=1$.

This property implies that the action is LS covariant. To see this consider the product

$$
\begin{aligned}
\mathcal{F}_{\text {can }}^{-} \phi \star \mathcal{F}_{\text {can }}^{+} \phi & =T\left(T^{-1} \mathcal{F}_{\text {can }}^{-} \phi \star_{\theta}^{0} T^{-1} \widetilde{F}_{\text {can }}^{+} \phi\right) \\
& =T\left(\mathcal{F}_{-\omega} T^{-1} \phi \star_{\theta}^{0} \mathcal{F}_{+} T^{-1} \phi\right) \\
& =T\left(T^{-1} \phi \star_{\theta}^{0} T^{-1} \phi\right)=\phi \star \phi .
\end{aligned}
$$

The same transformation for the quadratic part of the action holds, since

$$
\mathcal{F}_{\text {can }}^{-} \phi \star \Delta^{L S} \mathscr{F}_{\text {can }}^{+} \phi=\mathscr{F}_{\text {can }}^{-} \phi \star \mathscr{F}_{\text {can }}^{+} \Delta^{L S} \phi=\phi \star \Delta^{L S} \phi
$$

and $\mathscr{F}_{\text {can }}^{+}$is an involution. Note that the metric (derived from the Laplacian) is in these coordinates

$$
g^{\mu v}=\left(\begin{array}{cccc}
1 & -2 z & x^{1} & x^{2} \\
& 1+4 z^{2}+\frac{\left[\left(x^{1}\right)^{2}+\left(x^{2}\right)^{2}\right]}{4} & \frac{x^{2}}{2}-2 z x^{1} & -\frac{x^{1}}{2}-2 z x^{2} \\
& & 1+\left(x^{1}\right)^{2} & x^{1} x^{2} \\
& & & 1+\left(x^{2}\right)^{2}
\end{array}\right)
$$

where $\mu, v=a, z, x^{1}, x^{2}$.

4.2. Moyality principle. In order to carry out a study of the renormalizability of this theory we need to introduce the $p$-point kernel corresponding to a $p$-point vertex. To compute it, start from

$$
\operatorname{Tr}\left(f_{p} \star f_{p-1} \star \cdots \star f_{1}\right)=\int \prod_{i=1}^{p} d x_{i} \prod_{i=1}^{p} f\left(x_{i}\right) \operatorname{Tr}\left(\delta_{x_{p}} \star \delta_{x_{p-1}} \star \cdots \star \delta_{x_{1}}\right),
$$

where we have used $f(x)=\int d x_{1} f\left(x_{1}\right) \delta_{x_{1}}(x)$ and the linearity of the $\star$ and of the trace. The $p$ point vertex kernel is thus

$$
\mathbf{K}^{p}\left(x_{p}, \ldots, x_{1}\right)=\operatorname{Tr}\left(\delta_{x_{p}} \star \delta_{x_{p-1}} \star \cdots \star \delta_{x_{1}}\right) .
$$

Since the trace is cyclic so is the $p$-point kernel. Note that if the $\star$ product is tracial, the 3-point kernel is identical with the kernel of the product between (denoted $K(x, y, z))$ :

$$
\operatorname{Tr}\left(\delta_{x_{3}} \star \delta_{x_{2}} \star \delta_{x_{1}}\right)=\int d x \delta_{x_{3}}(x) \int d y d z K(x, y, z) \delta_{x_{2}}(y) \delta_{x_{1}}(z) .
$$


Let $G$ be a Feynman graph. Any reasonable scale behavior of the propagator will lead to locality in the ultraviolet region (as it behaves like a flat propagator). We will consider for the remainder of this section the limit in which the propagator is ultralocal, that is, $C(x, y)=\delta(x-y)$. Indeed at short distance this is expected to be the right approximation even in curved space to study the "Moyality" of the counterterms [35], [52]

In order to rewrite the vertex contribution of the graph we chose a rooted tree in the graph. We assign a total ordering of the tree lines corresponding to turning around the tree in the trigonometric way. We introduce the first topological operation, the tree line reduction. At any step we contract the lowest line (in the total ordering) $\ell=(i, j)$ connecting the root vertex $V$ at $i$ to another vertex $v$ at $j$.

To evaluate the integral corresponding to this tree line we cyclically turn the root vertex so that $i$ becomes the first point on $V$. We also turn $v$ so that $j$ becomes the last point on $v$. The integral associated to this line is

$$
\begin{aligned}
& \int d z d t \mathbf{K}^{p+1}\left(x_{p}, \ldots, x_{1}, z\right) \delta(z-t) \mathbf{K}^{q+1}\left(t, y_{q}, \ldots, y_{1}\right) \\
& =\int d z d t d u d v\left(\delta_{x_{p}} \star \cdots \star \delta_{x_{1}}\right)(u) \delta_{z}(u) \delta(z-t) \delta_{t}(v)\left(\delta_{y_{q}} \star \cdots \star \delta_{y_{1}}\right)(v) \\
& =\mathbf{K}^{p+q}\left(x_{p}, \ldots, x_{1}, y_{q}, \ldots, y_{1}\right),
\end{aligned}
$$

where we have used the associativity and the traciality of the product.

After reducing the tree we end up with only the root vertex with some points on the root still connected by loop propagators. This vertex is called the rosette of the graph. Denote the points on the rosette by $y_{p}, \ldots, y_{1}$. They divide in loop half lines $\left(y_{i}^{\ell}, y_{j}^{\ell}\right)=\ell$ and true external points $y^{e}$. We have thus proved

Lemma 4.1. The vertex contribution at the amplitude of a graph is given by

$$
A\left(y^{e}, \ldots, y^{e}\right)=\int \prod_{\ell} d y_{i}^{\ell} d y_{j}^{\ell} \delta\left(y_{i}^{\ell}-y_{j}^{\ell}\right) \mathbf{K}^{p}\left(y_{p}, \ldots, y_{1}\right),
$$

where $\mathbf{K}^{p}$ is the p-point kernel corresponding to the rosette.

We now define the second topological operation. Take a loop line $\ell^{\prime}$ on the rosette (if it exists) such that the two endpoints of the line are nearest neighbors on the rosette. We can cyclically permute the rosette to set the first two points to be the end points of the line. The contribution of such a configuration is

$$
\begin{aligned}
& \int d y_{1} d y_{2} \mathbf{K}^{p}\left(y_{p}, \ldots, y_{2}, y_{1}\right) \delta\left(y_{2}-y_{1}\right) \\
& =\int d y_{1} d y_{2} d u\left(\delta_{y_{p}} \star \cdots \star \delta_{y_{3}}\right)(u)\left(\delta_{y_{2}} \star \delta_{y_{1}}\right)(u) \delta\left(y_{2}-y_{1}\right) .
\end{aligned}
$$


Using the product 8 we have

$$
\begin{aligned}
& \int d y_{1} d y_{2}\left(\delta_{y_{2}} \star \delta_{y_{1}}\right)(u) \delta\left(y_{2}-y_{1}\right) \\
& =\int d y K(u, y, y) \\
& =\frac{1}{\theta^{\operatorname{dim} \$}} \int_{-\infty}^{\infty} d a_{y} d x_{y} d z_{y} \sqrt{\cosh \left[2\left(a_{u}-a_{y}\right)\right] \cosh \left[2\left(a_{y}-a_{u}\right)\right]} \\
& =\frac{1}{\theta^{\operatorname{dim} \$}} \int_{-\infty}^{\infty} d x_{y} \int_{-\infty}^{\infty} d z_{y} \int_{-\infty}^{\infty} d a_{y} \cosh \left(2 a_{y}\right)\left[\cosh \left(a_{y}\right)\right]^{\frac{\operatorname{dim} \mathbb{S}-2}{2}},
\end{aligned}
$$

where we translated $a_{y}$ by $a_{u}$. The above integral is an infinite constant.

Thus the second topological operation for a line $\ell^{\prime}$ such that its endpoints are neighbors on the rosette simply erases its endpoints on the rosette and multiplies the latter by this infinite constant.

For a planar graph with only one face broken by external points we can iterate this operation for all loop lines. We have thus

Lemma 4.2. The factor of a planar one broken face p point graph is the p point kernel times an infinite constant.

This is the precise meaning of the Moyality of the theory: an arbitrary planar one broken face graph can be renormalized (if needed) by a Moyal counterterm.

\section{Extended algebra and metaplectic construction}

The relevance of the Langmann-Szabo self-dual propagator and its spectral analysis underlying the vulcanization process within the flat context may be independently interpreted in terms of the classical metaplectic representation of the Heisenberg group. Indeed, we start by considering the (commutative or not) flat configuration space $\mathbb{R}^{d}:=\left\{\left(x^{1}, \ldots, x^{d}\right)\right\}$ as a Lagrangian subspace of a co-adjoint orbit $\mathcal{O} \simeq$ $T^{\star}\left(\mathbb{R}^{d}\right)$ of the Heisenberg group $\mathbf{H}_{d}$ in the dual $\mathfrak{h}_{d}^{\star}$ of its Lie algebra $\mathfrak{h}_{d}$. In this framework, a natural representation Hilbert space $\left(\mathscr{H}=L^{2}\left(\mathbb{R}^{d}\right), \rho_{\hbar}\right)$ is canonically defined (through Kirillov's theory) as well as the following quantization rule:

$$
\begin{gathered}
\mathrm{Op}: \mathcal{S}(\mathcal{O}) \rightarrow \mathscr{B}(\mathcal{H}): u \mapsto \mathrm{Op}(u), \\
\mathrm{Op}(u) \varphi:=j^{\star}\left(u \star_{\theta}^{0} \pi^{\star} \varphi\right),
\end{gathered}
$$

where $\pi: \mathcal{O}=T^{\star}\left(\mathbb{R}^{d}\right) \rightarrow \mathbb{R}^{d}$ denotes the natural projection, $j: \mathbb{R}^{d} \rightarrow T^{\star}\left(\mathbb{R}^{d}\right)$ the null section, $\star_{\theta}^{0}$ denotes the Weyl product on $\mathcal{O}=T^{\star}\left(\mathbb{R}^{d}\right)$ and $\mathcal{B}(\mathscr{H})$ denotes 
the bounded operators on $\mathscr{H}$. The symplectic linear group $\operatorname{Sp}(d, \mathbb{R})$ naturally acts by automorphisms on the Heisenberg group $\mathbf{H}_{d}$, inducing an action on $\mathfrak{h}_{d}^{\star}$ and on $\mathcal{O}$ by restriction. Note that the Moyal-Weyl product is characterized as the only star product on $\mathcal{O}$ that is invariant under the action of the linear canonical transformation group ${ }^{2}$ $\operatorname{Sp}(d, \mathbb{R}) \times \mathbf{H}_{d}$. The metaplectic group/representation can now be defined as follows [61]. One first observes that the Levy factor of the Lie algebra of derivations of $\mathfrak{h}_{d}$ that vanish on its center is isomorphic to the Lie algebra $\mathfrak{s p}(d, \mathbb{R})$ of $\operatorname{Sp}(d, \mathbb{R})$. The Stone-von Neumann theorem ${ }^{3}$ then implies that every element $A$ of the corresponding analytic subgroup of automorphisms of $\mathfrak{h}_{d}$ acts (up to a sign) on the Hilbert space $\mathscr{H}$ via the representation $\mu$ through the defining formula

$$
\mu(A) \circ \rho_{\hbar} \circ \mu\left(A^{-1}\right):=\rho_{\hbar} \circ A .
$$

It turns out that the vulcanized propagator $\Delta_{0}+\Omega^{2}\left|x^{2}\right|$ is nothing else than the operator in $\mathscr{H}$ associated to a specific element $Z_{\Omega}$ of $\mathfrak{s p}(d, \mathbb{R})$ by the infinitesimal metaplectic representation ([25], p. 186):

$$
\Delta_{0}+\Omega^{2}\left|x^{2}\right|=4 \pi i \mu_{\star e}\left(Z_{\Omega}\right)
$$

where

$$
Z_{\Omega}:=\left(\begin{array}{cc}
0 & \frac{\Omega^{2}}{8 \pi^{2}} I \\
I & 0
\end{array}\right)
$$

In particular, the spectral analysis of our vulcanized propagator can be performed purely in classical representation theoretical terms. For instance, Mehler's kernel can be simply derived from the expression of (analytic continuation) of $\mu \exp \left(t Z_{\Omega}\right)$.

Alternatively, in order to compute the operator semigroup $e^{t\left(\Delta_{0}+\Omega^{2}\left|x^{2}\right|\right)}$, one may also use the well-known explicit expressions for the Moyal star exponentials of quadratic Hamiltonians (see e.g. [48]). Indeed, extending the above quantization rule (23) to polynomial symbols the operator semigroup may be regarded as

$$
e^{t\left(\Delta_{0}+\Omega^{2}\left|x^{2}\right|\right)} \varphi=j^{\star}\left(e_{\theta}^{t \lambda Z_{\Omega}} \star_{\theta}^{0} \pi^{\star} \varphi\right),
$$

where $\lambda_{X}$ denotes the classical (quadratic) Hamiltonian function on $\mathcal{O}$ associated to the element $X \in \mathfrak{s p}(d, \mathbb{R})$ and where the star exponential is formally defined as

$$
e_{\theta}^{f}:=\sum_{k=0}^{\infty} \frac{1}{k !}\left(f \star_{\theta}^{0} \cdots \star_{\theta}^{0} f\right) \quad(k \text { times }) .
$$

\footnotetext{
${ }^{2}$ In particular, starting from a noncommutative configuration Moyal space $\mathbb{R}_{\theta}^{d}$ rather than a commutative one does not affect in any respect the present discussion.

${ }^{3}$ It characterizes a unitary irreducible representation of the Heisenberg group by its character of the center.
} 
We therefore observe that a natural geometrical framework to implement the Langmann-Szabo duality consists in implementing our situation within a purely representation theoretical context where the configuration space plays the role of polarized orbit. In view of passing to a general curved situation, a first essential ingredient is therefore a notion of 'extended' algebra that will play to role of the Heisenberg algebra in the flat case as discussed above. It turns out that the quantum moments $\rho_{v}(X)$ together with their LS-dual elements $\mathcal{F}_{+\omega} \rho_{v}(X) \mathcal{F}_{+\omega}(X \in \mathfrak{s})$ generate a finite dimensional Lie algebra.

Now, back to our curved situation, the consideration made in Section 3.3 naturally leads to considering the algebra generated by the following differential operators acting on $C^{\infty}(\widetilde{\Phi})$ :

$$
X^{0}:=\mathscr{F} \rho_{\nu}(X) \mathcal{F}^{-1} \quad \text { and } \quad X^{1}:=\mathscr{F}^{\mathcal{F}}+\omega \rho_{\nu}(X) \mathcal{F}_{+\omega} \mathscr{F}^{-1}, \quad X \in \mathfrak{s} .
$$

It turns out that the above operators generate a finite dimensional Lie algebra that consists in an analog of the Heisenberg algebra in the present curved context. Indeed, one first sets

$$
\begin{aligned}
w^{10} & :=-\theta e^{-a}\left(\sinh (\theta \xi) \partial_{w}+\frac{i}{\theta} \omega(v, w) \cosh (\theta \xi)\right) \quad(w \in V), \\
E^{01,1} & :=i e^{-2 \xi} \cosh (2 \theta a), \\
w^{01} & :=-\theta e^{-\xi}\left(i \sinh (\theta a) \omega(v, w)-\frac{1}{\theta} \cosh (\theta a) \partial_{w}\right) \quad(w \in V), \\
E^{10,0} & :=-i e^{-2 a} \cosh (2 \theta \xi), \\
E^{\epsilon, \epsilon^{\prime}} & :=\frac{i}{4 \theta^{2}}\left(\theta^{2}+\epsilon \epsilon^{\prime}\right) e^{\epsilon(\theta-\epsilon) \xi+\epsilon^{\prime}\left(\theta-\epsilon^{\prime}\right) a} \quad\left(\epsilon, \epsilon^{\prime} \in\{1,-1\}, \theta \neq 0\right) .
\end{aligned}
$$

One then computes:

$$
\begin{aligned}
& {\left[H^{0}, w^{1}\right]=w^{01}, \quad\left[H^{1}, w^{0}\right]=w^{10},} \\
& {\left[H^{0}, w^{10}\right]=w^{10}, \quad\left[w^{01}, v^{1}\right]=\omega(w, v) E^{01,1},} \\
& {\left[H^{0}, w^{01}\right]=\theta^{2} w^{1}, \quad\left[w^{10}, v^{0}\right]=\omega(w, v) E^{10,0},} \\
& {\left[H^{1}, w^{01}\right]=w^{01}, \quad\left[w^{10}, v^{10}\right]=-\theta^{2} \omega(w, v) E^{0},} \\
& {\left[H^{1}, w^{10}\right]=\theta^{2} w^{0}, \quad\left[w^{01}, v^{01}\right]=-\theta^{2} \omega(w, v) E^{1},} \\
& {\left[w^{0}, v^{1}\right]=\omega(w, v)\left(E^{++}+E^{--}+E^{+-}+E^{-+}\right),} \\
& {\left[H^{0}, E^{1}\right]=2 E^{01,1}, \quad\left[H^{1}, E^{0}\right]=-2 E^{10,0},} \\
& {\left[H^{0}, E^{01,1}\right]=2 \theta^{2} E^{1}, \quad\left[H^{1}, E^{01,1}\right]=2 E^{01,1} \text {, }} \\
& {\left[H^{0}, E^{10,0}\right]=2 E^{10,0}, \quad\left[H^{1}, E^{10,0}\right]=-2 \theta^{2} E^{0} \text {, }} \\
& {\left[H^{0}, E^{++}\right]=(1-\theta) E^{++}, \quad\left[H^{1}, E^{++}\right]=(1-\theta) E^{++} \text {, }}
\end{aligned}
$$




$$
\begin{array}{rlrl}
{\left[H^{0}, E^{+-}\right]} & =(1-\theta) E^{+-}, & & {\left[H^{1}, E^{+-}\right]=(1+\theta) E^{+-},} \\
{\left[H^{0}, E^{-+}\right]=(1+\theta) E^{-+},} & & {\left[H^{1}, E^{-+}\right]=(1-\theta) E^{-+},} \\
{\left[H^{0}, E^{--}\right]=(1+\theta) E^{--},} & & {\left[H^{1}, E^{--}\right]=(1+\theta) E^{--} .}
\end{array}
$$

From the above brackets, one observes that the Jacobi identity implies that the vector space

$$
\mathrm{g}^{\mathrm{ext}}:=\operatorname{span}\left\{X^{0}, X^{1}, w^{10}, w^{01}, E^{10,0}, E^{01,1}, E^{\epsilon, \epsilon^{\prime}}\right\}_{X \in \mathfrak{s}, w \in V}
$$

closes as a finite dimensional Lie algebra. Note the following:

- The structure of the Lie algebra $g^{\text {ext }}$ slightly depends on the value of the real parameter $\theta$.

- The structure equation above labeled by $(*)$ is actually not singular for the value zero of the parameter. Indeed, it may be re-expressed as

$$
\left[w^{0}, v^{1}\right]=\omega(w, v) i e^{-a-\xi}\left(\cosh (\theta a) \cosh (\theta \xi)+\frac{1}{\theta^{2}} \sinh (\theta a) \sinh (\theta \xi)\right) .
$$

- For generic values of $\theta$ and when $\operatorname{dim} \mathfrak{s} \geq 4$, the dimension is given by

$$
\operatorname{dim} g^{\mathrm{ext}}=4 \operatorname{dim} \mathfrak{s}+2=2(2 \operatorname{dim} \mathfrak{s}-1)+4=2 \operatorname{dim} g^{c}+4,
$$

where $\mathrm{g}^{c}$ denotes the transvection algebra of the contracted symmetric space $(\$, s)$.

- In the two-dimensional case $(\operatorname{dim} s=2)$, the Lie algebra structure degenerates as

$$
\begin{aligned}
& {\left[H^{0}, E^{1}\right]=2 E^{01,1}, \quad\left[H^{1}, E^{0}\right]=-2 E^{10,0},} \\
& {\left[H^{0}, E^{01,1}\right]=2 \theta^{2} E^{1}, \quad\left[H^{1}, E^{01,1}\right]=2 E^{01,1} \text {, }} \\
& {\left[H^{0}, E^{10,0}\right]=2 E^{10,0}, \quad\left[H^{1}, E^{10,0}\right]=-2 \theta^{2} E^{0} \text {. }}
\end{aligned}
$$

In the two-dimensional case the (6-dimensional) Lie algebra $g$ ext turns out to be isomorphic to the 'double' of the transvection algebra of the contracted symmetric space $(\mathbb{S}, \omega, s)$. Indeed, the parts $\left\{H^{0}, E^{1}, E^{01,1}\right\}$ and $\left\{H^{1}, E^{0}, E^{10,0}\right\}$ respectively generate supplementary subalgebras that are both isomorphic to $\mathrm{g}^{c}$. Moreover, $\mathrm{g}^{c}$ carries a natural symmetric space structure resembling to the classical 'exchange case' in the semisimple theory [4]:

$$
\sigma^{c}\left(H^{0}\right):=H^{1}, \quad \sigma^{c}\left(E^{0}\right):=E^{1}, \quad \sigma^{c}\left(E^{01,1}\right):=E^{10,0}, \quad\left(\sigma^{c}\right)^{2}:=\mathrm{id} .
$$

An analogous discussion can be performed in the general case. We postpone to a subsequent work the detailed analysis of the Kirillov unireps associated with the relevant co-adjoint orbits. 


\section{Conclusion}

We have defined the analog of the Grosse-Wulkenhaar model on SSS. The next steps should be to renormalize and explore the physics of these models. Renormalization requires:

- A "scale analysis" or more precisely a spectral analysis of the propagator, to define renormalization group steps.

The propagator $C=1 / Q$ is the inverse of the quadratic part of the action $Q$. The Schwinger representation $C=\int_{0}^{\infty} e^{-t Q} d t$ is convenient to define a geometric series of scales such as $C=\sum C^{i}, C^{i}=\int_{M^{-i}}^{M^{-(i-1)}} e^{-t Q} d t$. In an appropriate representation one should derive explicit bounds that capture both the short distance behavior (governed by the Laplacian hence expected similar to the heat kernel behavior of ordinary commutative space) and the long distance behavior that depends on the vulcanization terms. This representation could be either direct space, coherent states (also called "matrix representation") or some mixed representation like the partial Fourier transform used in (6) and Proposition 3.3.

- These bounds should be combined with the vertex kernel (computed in the same representation) in order to establish power counting for Feynman graphs. This power counting is dimension- and model-dependent. In dimension four the theory should be renormalizable. This means that the only amplitudes which diverge when the difference between their internal and external scales increases can be written as a local part of the form of the initial action plus a remainder which no longer diverges. For the coupling constant and mass renormalization this step should be a direct consequence of the scale analysis and of the Moyality principle of Section 4.2. However the so-called wave function renormalization is a bit more subtle. It requires an extension of that Moyality principle to "second order" for the two point function. Indeed beyond the zeroth order which corresponds to mass renormalization one needs to prove that the second order approximation recovers exactly the coefficients of the vulcanized propagator, namely renormalizes the coefficient of the Laplacian (this is usually absorbed in a rescaling of the field variable) and the coefficient of the vulcanization term.

- Renormalization flows should be computed to check whether absence of Landau ghosts survive on these non flat SSS backgrounds. If this is the case (as we a priori expect) the non perturbative construction of the theory should follow.

This renormalization program and the mathematical relation of LS duality to metaplectic representations will be explored in future publications. 


\section{References}

[1] J. Ben Geloun and V. Rivasseau, Color Grosse-Wulkenhaar models: One-loop $\beta$ functions. Preprint 2008. arXiv:0805.2538

[2] J. Ben Geloun, R. Gurau, and V. Rivasseau, Vanishing beta function for GrosseWulkenhaar model in a magnetic field. Preprint 2008. arXiv:0805.4362

[3] F. A. Berezin, Quantization in complex symmetric spaces. Izv. Akad. Nauk SSSR Ser. Mat. 39 (1975), 363-402; English transl. Math. USSR-Izv. 9 (1975), 341-379. Zbl 0324.53049 MR 0508179

[4] M. Berger, Les espaces symétriques non compacts. Ann. Sci. École Norm. Sup. (3) 74 (1957), 85-177. Zbl 0093.35602 MR 0104763

[5] P. Bieliavsky, Espaces symetriques symplectiques. Ph. D. thesis, Université Libre de Bruxelles, 1995. arXiv:math/0703358

[6] P. Bieliavsky, Strict quantization of solvable symmetric spaces. J. Symplectic Geom. 1 (2002), 269-320. Zbl 1032.53080 MR 1959584

[7] P. Bieliavsky, Deformation quantization for actions of the affine group. Preprint 2007. arXiv:0709.1110

[8] P. Bieliavsky, Non-formal deformation quantizations of solvable Ricci-type symplectic symmetric spaces. Preprint 2007. arXiv:0711.4002

[9] P. Bieliavsky, P. Bonneau, and Y. Maeda, Universal deformation formulae, symplectic Lie groups and symmetric spaces. Pacific J. Math. 230 (2007), 41-57. MR 2318447

[10] P. Bieliavsky, M. Cahen, and S. Gutt, A class of homogeneous symplectic manifolds. In Geometry and nature (Madeira, 1995), Contemp. Math. 203, Amer. Math. Soc., Providence, RI, 1997, 241-255. Zbl 0879.53026 MR 1443929

[11] P. Bieliavsky, L. Claessens, D. Sternheimer and Y. Voglaire, Quantized anti de Sitter spaces and non-formal deformation quantizations of symplectic symmetric spaces. In Poisson geometry in mathematics and physics (Tokyo, 2006), Contemp. Math. 450, Amer. Math. Soc., Providence, RI, 2008, 1-24. Zbl 05310305

[12] P. Bieliavsky, S. Detournay, P. Spindel, and M. Rooman, Star products on extended massive non-rotating BTZ black holes. J. High Energy Phys. 06 (2004), 031. Zbl MR 2084424

[13] P. Bieliavsky and M. Massar, Oscillatory integral formulae for left-invariant star products on a class of Lie groups. Lett. Math. Phys. 58 (2001), 115-128. Zbl 0998.53059 MR 1876248

[14] P. Bieliavsky and M. Massar, Strict deformation quantization for actions of a class of symplectic Lie groups. Progr. Theoret. Phys. Suppl., no. 144 (2001), 1-21. Zbl 1018.53044 MR 2023842; http://ptp.ipap.jp/link?PTPS/144/1/

[15] A. H. Chamseddine and A. Connes, The spectral action principle. Comm. Math. Phys. 186 (1997), 731-750. Zbl 0894.58007 MR 1463819

[16] A. H. Chamseddine, A. Connes, and M. Marcolli, Gravity and the standard model with neutrino mixing. Adv. Theor. Math. Phys. 11 (2007), 991-1089. Zbl 1140.81022 MR 2368941 
[17] A. Connes, Noncommutative geometry. Academic Press, San Diego 1994.Zbl 0818.46076 MR 1303779

[18] A. Connes, Gravity coupled with matter and the foundation of non-commutative geometry. Comm. Math. Phys. 182 (1996), 155-176. Zbl 0881.58009 MR 1441908

[19] A. Connes and J. Lott, Particle models and noncommutative geometry. Nuclear Phys. B Proc. Suppl. 18B (1990), 29-47. Zbl 0957.46516 MR 1128127

[20] A. de Goursac, A. Tanasa, and J.-C. Wallet, Vacuum configurations for renormalizable noncommutative scalar models. Eur. Phys. J. C Part. Fields 53 (2008), 459-466. MR 2367062

[21] A. de Goursac, J.-C. Wallet, and R. Wulkenhaar, Noncommutative induced gauge theory. Eur. Phys. J. C Part. Fields 51 (2007), 977-987. MR 2336186

[22] M. Disertori, R. Gurau, J. Magnen, and V. Rivasseau, Vanishing of beta function of noncommutative $\Phi_{4}^{4}$ theory to all orders. Phys. Lett. B 649 (2007), 95-102. Zbl MR 2308801

[23] M. Disertori and V. Rivasseau, Two- and three-loop beta function of non-commutative $\phi_{4}^{4}$ theory. Eur. Phys. J. C 50 (2007), 661-671.

[24] T. Filk, Divergencies in a field theory on quantum space. Phys. Lett. B 376 (1996), 53-58. MR 1395558

[25] G. B. Folland, Harmonic analysis in phase space. Ann. of Math. Stud. 122, Princeton University Press, Princeton, NJ, 1989. Zbl 0682.43001 MR 0983366

[26] V. Gayral, Heat-kernel approach to UV/IR mixing on isospectral deformation manifolds. Ann. Henri Poincaré 6 (2005), 991-1023. Zbl 1082.58006 MR 2189376

[27] H. Grosse and H. Steinacker, A nontrivial solvable noncommutative $\phi^{3}$ model in 4 dimensions. J. High Energy Phys. 08 (2006), 008. MR 2249954

[28] H. Grosse and H. Steinacker, Renormalization of the noncommutative $\phi^{3}$ model through the Kontsevich model. Nuclear Phys. B 746 (2006), 202-226. Zbl 05151221 MR 2235178

[29] H. Grosse and H. Steinacker, Exact renormalization of a noncommutative $\phi^{3}$ model in 6 dimensions. Adv. Theor. Math. Phys. 12 (2008), 605-639. Zbl 05312054 MR 2399320

[30] H. Grosse and M. Wohlgenannt, Induced gauge theory on a noncommutative space. Eur. Phys. J. C Part. Fields 52 (2007), 435-450. MR 2346157

[31] H. Grosse and R. Wulkenhaar, The $\beta$-function in duality-covariant non-commutative $\phi^{4}$-theory. Eur. Phys. J. C Part. Fields 35 (2004), 277-282. MR 2075076

[32] H. Grosse and R. Wulkenhaar, Power-counting theorem for non-local matrix models and renormalisation. Comm. Math. Phys. 254 (2005), 91-127. Zbl 1079.81049 MR 2116739

[33] H. Grosse and R. Wulkenhaar, Renormalization of $\phi^{4}$-theory on noncommutative $\mathbb{R}^{4}$ in the matrix base. Commun. Math. Phys. 256 (2005), 305-374. Zbl 1075.82005 MR 2160797

[34] H. Grosse and R. Wulkenhaar, 8D-spectral triple on 4D-Moyal space and the vacuum of noncommutative gauge theory. Preprint 2007. arXiv:0709.0095

[35] R. Gurau, J. Magnen, V. Rivasseau, and F. Vignes-Tourneret, Renormalization of noncommutative $\Phi_{4}^{4}$ field theory in $x$ space. Comm. Math. Phys. 267 (2006), 515-542. Zbl 1113.81101 MR 2249779 
[36] R. Gurau, A. Malbouisson, V. Rivasseau, and A. Tanasa, Non-commutative complete Mellin representation for Feynman amplitudes. Lett. Math. Phys. 81 (2007), 161-175. MR 2336230

[37] R. Gurau and V. Rivasseau, Parametric representation of noncommutative field theory. Comm. Math. Phys. 272 (2007), 811-835. Zbl 05202353 MR 2304476

[38] R. Gurau, V. Rivasseau, and F. Vignes-Tourneret, Propagators for noncommutative field theories. Ann. Henri Poincaré 7 (2006), 1601-1628. Zbl 1110.81138 MR 2283747

[39] R. Gurău and A. Tanasă, Dimensional regularization and renormalization of noncommutative quantum field theory. Ann. Henri Poincaré 9 (2008), 655-683. Zbl 05303978 MR 2413199

[40] W. Heisenberg, Die Grenzen der Anwendbarkeit des bisherigen Quantentheorie. Z. Phys. 110 (1938), 251-266. JFM 64.0890.01 Zbl 0021.27506

[41] S. Hellerman and M. Van Raamsdonk, Quantum Hall physics equals noncommutative field theory? J. High Energy Phys. 10 (2001), 039. MR 1877548

[42] A. Lakhoua, F. Vignes-Tourneret, and J. Wallet, One-loop beta functions for the orientable non-commutative Gross-Neveu model. Preprint 2007. arXiv:hep-th/0701170

[43] E. Langmann and R. J. Szabo, Duality in scalar field theory on noncommutative phase spaces. Phys. Lett. B $\mathbf{5 3 3}$ (2002), 168-177. Zbl 0994.81116 MR 1911944

[44] E. Langmann, R. J. Szabo, and K. Zarembo, Exact solution of noncommutative field theory in background magnetic fields. Phys. Lett. B 569 (2003), 95-101. Zbl 1059.81608 MR 2044143

[45] E. Langmann, R. J. Szabo, and K. Zarembo, Exact solution of quantum field theory on noncommutative phase spaces. J. High Energy Phys. 01 (2004), 017. MR 2045889

[46] J. Magnen and V. Rivasseau, Constructive $\phi^{4}$ field theory without tears. Ann. Henri Poincaré 9 (2008), 403-424. Zbl 1141.81022 MR 2399194

[47] A. Matusis, L. Susskind, and N. Toumbas, The IR/UV connection in non-commutative gauge theories. J. High Energy Phys. 12 (2000), 002. Zbl 0990.81549 MR 1813360

[48] H. Omori, Y. Maeda, N. Miyazaki, and A. Yoshioka, Star exponential functions for quadratic forms and polar elements. In Quantization, Poisson brackets and beyond (Manchester, 2001), Contemp. Math. 315, Amer. Math. Soc., Providence, RI, 2002, 25-38. Zbl 1047.53057 MR 1958828

[49] A. P. Polychronakos, Quantum Hall states as matrix Chern-Simons theory. J. High Energy Phys. 04 (2001), 011. MR 1834406

[50] M. A. Rieffel, Deformation quantization of Heisenberg manifolds. Comm. Math. Phys. 122 (1989), 531-562. Zbl 0679.46055 MR 1002830

[51] V. Rivasseau, Constructive matrix theory. J. High Energy Phys. 09 (2007), 008. MR 2342423

[52] V. Rivasseau, Non-commutative renormalization. In Quantum spaces: Poincaré Seminar 2007, Prog. Math. Phys. 53, Birkhäuser, Basel 2007, 19-107. Zbl 1139.81047 MR 2382235 
[53] V. Rivasseau and A. Tanasă, Parametric representation of "critical" noncommutative QFT models. Preprint 2007. arXiv:math-ph/0701034

[54] V. Rivasseau, F. Vignes-Tourneret, and R. Wulkenhaar, Renormalisation of noncommutative $\phi^{4}$-theory by multi-scale analysis. Comm. Math. Phys. 262 (2006), 565-594. Zbl 1109.81056 MR 2202303

[55] E. Schrödinger, Über die Unanwendbarkeit der Geometrie im Kleinen. Naturwiss. 22, no. 31 (1934), 518-520. JFM 60.1431.01 Zbl 0009.38101

[56] K. Sekigawa and L. Vanhecke, Symplectic geodesic symmetries on Kähler manifolds. Quart. J. Math. Oxford Ser. (2) 37 (1986), 95-103. Zbl 0589.53068MR 830633

[57] H. S. Snyder, Quantized space-time. Phys. Rev. (2) 71 (1947), 38-41. Zbl 0035.13101 MR 0019438

[58] L. Susskind, The quantum Hall fluid and non-commutative Chern Simons theory. Preprint 2001. arXiv:hep-th/0101029

[59] A. Tanasă and F. Vignes-Tourneret, Hopf algebra of non-commutative field theory. J. Noncommut. Geom. 2 (2008), 125-139. Zbl 05247285 MR 2366125

[60] A. Unterberger and J. Unterberger, Quantification et analyse pseudo-différentielle. Ann. Sci. École Norm. Sup. (4) 21 (1988), 133-158. Zbl 0646.58025 MR 0944104

[61] L. Van Hove, Sur certaines représentations unitaires d'un groupe infini de transformations. Acad. Roy. Belg. Cl. Sci. Mém. Collect. $8^{\circ}$ (2) 26, no. 6 (1951). Zbl 0045.38701 MR 0057260

[62] F. Vignes-Tourneret, Renormalisation des théories de champs non commutatives. Ph. D. thesis, Université Paris 11, 2006. arXiv:math-ph/0612014

[63] F. Vignes-Tourneret, Renormalization of the orientable non-commutative Gross-Neveu model. Ann. Henri Poincaré 8 (2007), 427-474. Zbl 1133.81058 MR 2329358

[64] Z. Wang and S. Wan, Renormalization of orientable non-commutative complex $\Phi_{3}^{6}$ model. Ann. Henri Poincaré 9 (2008), 65-90. Zbl 1142.81019 MR 2389890

[65] A. Weinstein, Traces and triangles in symmetric symplectic spaces. In Symplectic geometry and quantization (Sanda and Yokohama, 1993), Contemp. Math. 179, Amer. Math. Soc., Providence, RI, 1994, 261-270. Zbl 0820.58024 MR 1319613

Received June 26, 2008

P. Bieliavsky, Département de Mathématiques, Université Catholique de Louvain, Bât. M de Hemptinne, Chemin du Cyclotron, 2, 1348 Louvain-La-Neuve, Belgium E-mail: bieliavsky@math.ucl.ac.be

R. Gurau, V. Rivasseau, Laboratoire de Physique Théorique, CNRS UMR 8627, Bât. 210, Université Paris XI, 91405 Orsay Cedex, France

E-mail: razvan.gurau@th.u-psud.fr; rivass@th.u-psud.fr 